\title{
Bericht über die XIX. Jahrestagung der Gesellschaft für Afrikanisches Recht e.V. in Heidelberg am 5. und 6. November 1993
}

\author{
Von Harald Sippel
}

Am 5. und 6. November 1993 fand im Heidelberger Max-Planck-Haus die neunzehnte Jahrestagung der Gesellschaft für Afrikanisches Recht statt. Uber 30 Wissenschaftlerinnen und Wissenschaftler aus sieben Ländern folgten der Einladung des Vorstandsvorsitzenden der Gesellschaft, Herm Prof. Dr. Ulrich Spellenberg von der Universität Bayreuth, an den Vorträgen der drei afrikanischen Juristen, die das Programm der Jahreskonferenz 1993 vorsah, und den sich daran anschließenden Diskussionen teilzumehmen.

Zunächst begann Hers Dr. Cyprian F. Fisiy von der Universität von Yaoundé in Kamerun, gegenwärtig an der Landwirtschaftlichen Universität Wageningen/Niederlande am Institut für Agrar-Recht tätig, mit seinem Konferenzbeitrag über "Chieftaincy in the Modem State: An Institution at the Crossroads of Democratic Change".1

Der Referent leitete seinen Vortrag mit der Fordenung ein, daB Afrika seine politischen Institutionen demokratisieren müsse. Am Beispiel der Nordwest-Provinz von Kamerun fühne er aus, wie es die dortigen "Häuptlinge" verstanden hätten, während der Kolonialzeit sowie der nachfolgenden Unabhängigkeitsphase durch ein Zusammenspiel von Ritualen, Mythen, Allianzen sowie politischen Winkelzügen die Kontrolle über die Bevölkerung zu behalten, und welche Aufgaben ihnen im Rahmen des Demokratisierungsprozesses zufallen könnten. Dabei ging er insbesondere von der Fragestellung aus, welche Rolle der Institution des nach westlichem Demokratie-Verständnis anachronistisch anmutenden "Häuptlings" als "vote bank" beigemessen werden kann, d.h. in welchem $\mathrm{Ma} \beta$ dem Fon ein bestimmender Einfluß auf das Stimmverhalten des durch ihn traditionell repräsentierten Volkes bei demokratischen Wahlen zukommen kann.

Die in der Nordwest-Provinz von Kamerun gemeinhin als Fons bezeichneten "Häuptlinge" hätten in der Vergangenheit über eine überragende Führungsposition verfügt, deren Legitimation mythischen Ursprungs sei. Legendenbildungen hätten in der traditionellen Gesell-

1 Die gegenwärtige politische und gesellschaftliche Stellung der traditionellen "Häupelinge" im modemen af rikanischen Nationalstaat eff reut sich in neuester Zeit zunehmender Auf merksamkeit; vgl. hierzu meinen Tagungsbericht in: Verf assung und Recht in Übersee 26 (1993), S. 212-216 (214). 
schaft den Eindruck hinterlassen, daß die allgewaltigen Gottheiten einschließlich der Erdgötter für die Belange der Menschen nur über den Fon zugänglich seien. Aus diesem Grunde traten die Fons als Vermittler zwischen dem Volk und den metaphysischen Kräften auf. Dabei hätten sie es verstanden, ihren an das Volk gerichteten Eingebungen einen wirrdigen zeremoniellen Rahmen zu verleihen, wofür ihnen im Gegenzug die allgemeine Ehrerbietung durch die Bevölkenung dargebracht worden sei. Mythos und Ritual hätten daher auch ihrer Herrschaftsausübung einen übersinnlichen Gehalt gegeben, der sie als anerkannte Autoritäten bestätigte. Diese Stellung sei ihnen nach der Unabhängigkeit Kamenus von der jungen politischen Elite des Landes streitig gemacht worden, die infolge der traditionellen Machtverhältnisse bislang kaum Möglichkeiten gehabt hätte, an der Herrschaft zu partizipieren. Dank der teils im Ausland erworbenen Bildung sei es der neuen politischen Schicht gelungen, Positionen zu besetzen, aufgnund derer sie die Machtbefugnisse und das Ansehen der Fons in der Gesellschaft herabzusetzen versuchten. Als anschauliches Beispiel für die geänderte Lage erläuterte der Vortragende die Rolle der "Häuptlinge" bei der Landverteilung. Während dem Fon früher aufgnund seiner Verbindung zu den metaphysischen Erdgöttem die alleinige Entscheidungsbefugnis daruber zugestanden habe, wem er für welchen Zeitraum welches Stück Land gegen eine Tributleistung zur Bearbeitung zuwies, sei es schrittweise dazu gekommen, daß nunmehr staatliche Verwaltungsstellen über die Landverteilung, bei blober Beteiligung des jeweiligen Fon, befinden. Mit dieser Maßnahme sei bezweckt worden, durch Marginalisienung und Einbindung des traditionellen "Häuptlings" in staatliche Aufgaben die Autorität desselben zu untergraben, seine wichtigste Einkommensquelle versiegen zu lassen und im Volke den Glauben an die religiöse Verbundenheit zum Boden zu erschüttern.

Die "Häuptlinge" häıten auf diesen Machtverlust zum Teil mit Maßnahmen reagiert, die sowohl gegen die nationale Gesetzgebung als auch gegen gewohnheitsrechtliche Normen verstießen, indem sie beispielsweise traditionsgemäß unveräußerliches Land sogar an Gebietsfremde verkauften, um sich kurzfristig wirtschaftliche Vorteile zu verschaffen, bevor es zu weiteren Machtbeschneidungen durch die Regierungsstellen komme. Im weiteren hätten es die "Häuptlinge" verstanden, die Bevölkerung davon zu überzeugen, daß es auch im Falle der behördlichen Übertragung von Landtiteln der Versöhnung des neuen Landbesitzers bzw. Grundeigentümers mit den Erdgöttern bedürfe. Eine solche geschehe durch die Zustimmung des Fon zum Vertragsschluß gegen eine Geldzahlung oder sonstige materielle Gefälligkeiten, die im Werte ungefähr den früheren Tributleistungen entsprächen.

Die schleichende Korruption, die einseitige Zusammenarbeit mit politischen Parteien sowie der Egoismus der "Häuptlinge" führten nach Auffassung des Referenten dazu, da $B$ der Fon als Agent des Staates angesehen und seine althergebracht unumstrittene Führungsrolle von der Bevölkerung nicht mehr einhellig anerkannt werde. Abschließend bezweifelte der Vortragende daher die Akzeptanz des Fon als mögliche "vote bank" durch das von ihm traditionell repräsentierte Volk. 
In der sich anschließenden lebhaften Diskussion wurde insbesondere von den afrikanischen Teilnehmem die politische Stellung der traditionellen "Häuptlinge" im nordwestlichen Kamerim mit der auf einer anders verlaufenen historischen Entwicklung beruhenden abweichenden Lage in Nigeria und Tansania verglichen. Im weiteren wurden die gegenwärtig noch bestehenden oder angemaßten Befugnisse zur Ausübung der Straf- und Zivilgerichtsbarkeit auf gewohnheitsrechtlicher Grundlage durch die "Häuptlinge" in NordwestKamerım erörtert.

Mit dem Vortrag von Herm Ambroise K. Koffi Afande von der Juristischen Fakultät der Universität Lomé in Togo, gegenwärtig als Doktorand mit dem Max-Planck-Institut für internationales und ausländisches Strafrecht in Freiburg zusammenarbeitend, zu dem Thema "Das togolesische Straßenkind: An der Kreuzung von Recht und Rechtlosigkeit", wurde die Konferenz fortgeführt.

Der Referent wies einfuhrend auf das bereits allgemein bekannte Phänomen der auf der Sraße lebenden Kinder und Jugendlichen in südamerikanischen Städten hin, machte jedoch zugleich darauf aufmerksam, daß die sogenannten Straßenkinder zunehmend auch auf dem afrikanischen Kontinent, wie etwa in Togo, in Erscheinung träten. Als togolesisches Sraßenkind definierte der Vortragende ein Kind, das auf der Straße Tätigkeiten aller Art ausübt, die spielerischer Natur, auf Gewinnerzielung gerichtet, erlaubt oder verboten sein können, einerlei ob ihm die Möglichkeit verbleibt, an den heimatlichen Herd zurückzukehren oder nicht. Die Gründe für die Entstehung des Phänomens der Straßenkinder als bekannt voraussetzend, wies er im folgenden auf die Möglichkeiten zur Verbesserung der Lage dieses Personenkreises und dessen reale Lebensbedingungen in Togo hin, woran sich seine Beurteilung der vorgeschlagenen Maßnahmen anschloß.

Der Vortragende erörterte zunächst die möglichen Vorkehrungen zur Verbesserung der wirtschaftlichen und sozialen Situation des Straßenkinds, die auf eine erzieherische Unterstützung hinauslaufen. Die Vorschläge würden vorwiegend auf eine schulische, handwerkliche oder landwirtschaftliche Ausbildung des Straßenkindes abzielen und seine Rückkehr in seine Heimarregion anstreben. Dabei sollten die vorgesehenen Maßnahmen nicht gegen sondern vielmehr in Zusammenarbeit mit den betroffenen Kindern im Rahmen einer erzieherischen Aktion im offenen Umfeld (A.E.M.O., action éducative en milieu ouvert) ohne Unterbringung in geschlossenen Anstalten durchgeführt werden. Eine solche Ausgestaltung der Erziehung habe den Vorteil, daB sie billiger sei als eine Ausbildung im geschlossenen Umfeld. Außerdem bliebe dem Sraßenkind damit eine Internierung und "Ghettoisierung" infolge gemeinschaftlichen Wohnens mit anderen Kindern aus demselben Milieu erspart.

Im weiteren ging der Referent auf mögliche Ansätze zur Verbessenung der Lage des Straßenkindes durch die Sensibilisierung der Allgemeinheit und durch Reformansätze ein. Er führte aus, daß die togolesische Gesellschaft zunächst dazu gebracht werden müßte, daß sie ihren Teil der Verantwortung für den unglücklichen Zustand des Straßenkindes anzuerkennen habe, um dessen Kriminalisienung abzuwenden sowie den $\mathrm{HaB}$ und die Unterdrückung, die diesem entgegenschlagen, zu entschärfen. Diese Sensibilisienung der Gesell- 
schaft könnte nach Auffassung des Vortragenden einerseits durch sportliche oder kulturelle Aktivitäten der Straßenkinder selbst, andererseits durch Öfentlichkeitsarbeit mittels Konferenzen und der Massenmedien erreicht werden.

Anschließend zeigte er mögliche Reformansätze auf, die auf die weitgehende Legalisierung der von den Straßenkindem ausgeübten Kleingewerbe (petits-métiers) der Straße, zumeist in der Form von Handlanger-, Vermittlungs-, Händler- und Glücksspieltätigkeiten sowie Taschenspielerkunststücken auftretend, und auf eine Verändenung der Haltung von Polizei und Justiz gegenüber den Straßenkindem abstellen.

Nach der Darlegung der potentiellen Maßnahmen zur Verbesserung der Lage der Straßenkinder schilderte der Referent die gegenwärtig bestehenden Lebensbedingungen eines Straßenkindes in Togo. Diese seien von den unsicheren Verdiensimöglichkeiten, dem Verfall der Einnahmen und den drohenden vielfältigen Schicksalsschlägen bestimmt. Die unstetige Erwerbstätigkeit beruhe hauptsächlich auf der prekären Konkurrenzsituation zwischen den erwachsenen und jugendlichen Kleingewerbetreibenden sowie auf dem Kapitalmangel, während die schwindenden Einkunfte eine Folge der wirtschaftlichen Krise und der hohen Inflation in Togo seien. Das durchschnittliche Tageseinkommen eines togolesischen Straßenkindes entspräche derzeit lediglich einem Gegenwert von etwa 0,40 bis 0,50 DM. Für diesen spärlichen Lohn arbeite das Straßenkind oft bis zur körperlichen Erschöpfung, und nach Feststellungen des Referenten wüßten viele Arbeitgeber diesen ökonomischen Vorteil für sich nutzbar zu machen. Die Straßenkinder seien in Togo insoweit recht- und schutzlos einer Ausbeutung ausgesetzt, an der die Öfentlichkeit keinerlei Anteil nehme.

Recht- und schutzlos selbst innerhalb der eigenen sozialen Gruppe müsse das Straßenkind stets gewaltätige Ubergriffe und den Verlust seines armseligen Eigentums befürchten. Nach den Untersuchungen des Vortragenden sind Akte der Selbstjustiz von togolesischen Bürgern gegenüber Straßenkindem sowie unkoordinierte und willkürliche Massenverhaftungen derselben durch die Polizei mit anschließenden körperlichen und seelischen Mißhandlungen keine Seltenheit. Die Straftaten der Straßenkinder ahnde die Justiz mit Strafen, die im krassen Mißverhältnis zur Tat und zur Beute stünden. Es liege daher die Vermutung nahe, daß der togolesische Staat soziale Probleme mit kriminalpolitischen Mitteln zu lösen versuche. Da die gesetzgebende Gewalt in Togo die nötigen Reformen verschleppe, würden die staatlichen Stellen der Kinder- und Jugendfürsorge auf das Phänomen der Straßenkinder regelmäßig mit der Einweisung derselben in geschlossene Erziehungsanstalten reagieren.

Der Referent beendete seine Ausfühnmgen mit einem Vergleich der vorgeschlagenen Maßnahmen zur Verbesserung der wirtschaftlichen und sozialen Situation des Straßenkindes und seinen realen Lebensbedingungen in Togo. Dabei kam er zum Ergebnis, daß die theoretische Bestätigung der Vorrangigkeit des Erziehungsmodells im offenen Milieu (A.E.M.O.) in Togo keine effektive praktische Umsetzung erfahren habe. Er forderte, daß die togolesische Offentlichkeit vor allem durch einen verstärkten Einsatz der Medien für die besondere Situation der Straßenkinder sensibilisiert werden müsse. Schließlich solle der 
Staat endlich die versprochenen Gesetzesreformen zur Legalisienung der kleingewerblichen Tätigkeiten der Straßenkinder sowie zur Regelung der Kompetenzen der einzelnen Institutionen, die mit der Behandlung der Strabenkinder befaßt sind, erlassen. SchluBfolgernd stellte det Rednet fest, daß das togolesische Straßenkind gegenwärtig keinerlei Rechte besitze, bis auf das Recht, kein Recht zu haben.

Im AnschluB an den Vortrag wurden Detailfragen über die Herkunft und das Alter der togolesischen Straßenkinder sowie die Motive für ihre Beschäftigungen erörtert. Dabei wurde deutlich, daß das Phănomen der Straßenkinder in Togo nicht nur in der Metropole Lomé, sondern auch in den Kleinstädten und sogar in größeren Dörfern bestehe. Zur Frage hinsichtlich der Einbindung der Straßenkindet in die jüngsten innenpolitischen Konflikte in der Republik Togo erklärte der Referent, daß eine solche zwar vereinzelt stattgefunden habe, jedoch die Mehrheit der Kinder sich eher unpolitisch verhalte. Die aus der Mitte des Publikums angefragten konkreteren Lossungsvorschläge zur Verbesserung der Lage des togolesischen Straßenkindes blieb der Redner bislang freilich noch schuldig und verwies insoweit auf eine von ihm beabsichtigte größere Studie zu dieser Thematik.

Die Tagung wurde am Morgen des 6. November mit dem Referat von Herm Sengondo Mvungi von der Juristischen Fakultăt der Universitł̆ Dar es Salaam in Tansania, derzeit als Doktorand am Institut für Internationale Angelegenheiten der Universität Hamburg tätig, zu dem Thema "Towards the Law of Southem African Development Community (SADC)" fortgesetzt.

Der Vortragende wies zunächst auf die aus den Befreiungskämpfen der Völker im südlichen Afrika gegen die politische und wirtschafuliche Vorherrschaft der Republik Südafrika etklärbare Entstehungsgeschichte der Southern African Development Co-ordination Conference (SADCC) und die große ethnische, ökonomische und kulturelle Heterogenität der an ihr beteiligten zehn Staaten (Angola, Botswana, Lesotho, Malawi, Mozambique, Swaziland, Tansania, Zambia, Zimbabwe und seit kurzem auch Namibia) hin. Die SADCC hätte insbesondere die Ziele angestrebt, die ökonomische Abhängigkeit der Mitgliedsstaaten von der Republik Südafrika und anderen Gebieten der Erde zu reduzieren, die Zusammenarbeit untereinander zu fördem und gemeinsame Strategien zur intemationalen wirtschaftlichen Kooperation zu entwickeln. Dabei habe bei der Gritndung der SADCC im Jahre 1980 der Gedanke vorgeherrscht, daB, ăhnlich wie bei der Europäischen Gemeinschaft, über das Vehikel der wirtschaftlichen Integration langfristig eine politische Union der beteiligten Staaten geschaffen werden könne. Für die Durchführung eines solchermaßen bedeutsamen Vorhabens habe sich seinerzeit die Organization of African Unity (OAU) noch nicht entscheiden können. So hätten sich erst vor kurzem die Mitglieder det OAU zum AbschluB eines Vertrages entschlossen, wonach die Unterzeichnerstaaten innerhalb von 33 Jahren die Voraussetzungen für eine African Economic Community schaffen sollen, der von seiner Ausgestaltung und von seiner zeitlichen Dimension her jedoch mit den Vorhaben der SADCC nicht vergleichbar wäre. 
Der Referent fuhr fort mit der Erörtenung der rechtlichen Verbindlichkeit sowie der völkerrechtlichen Qualität der SADCC-Vereinbarungen, wonach die Gründungs-Deklaration der SADCC, die sogenannte Lusaka Declaration vom 1. April 1980, nur eine unverbindliche Absichtserklärung und nicht etwa ein völkerrechtlicher Vertrag zwischen den beteiligten Staaten sei. Aufgrund dieser informellen Organisationsstruktur erinnerten die SADCCMitglieder nach Ansicht des Vortragenden eher an einen bloßen "Club of Gentlemen". An diesem Umstand könne auch das "Memorandum of Understanding on the Institutions of the Southem African Development Co-ordination Conference" nichts ändem, das am 20. Juli 1981 zur Auslegung der Lusaka-Deklaration erging. Eine Ändenung der Situation sei erfolgt, als sich die Staatsoberhäupter der Mitgliedsländer der SADCC in Windhoek trafen, um dort am 17. August 1992 einen Vertrag zu unterzeichnen, durch den die SADCC aufgelöst wurde und die SADC als neue Organisation entstand. Dies sei ein bedeutender historischer Moment für die Völker im südlichen Afrika gewesen, weil die Mitgliedsstaaten durch die Formierung des neuen Bundes zu erkennen gegeben hätten, daB sie es nunmehr nach der zehnjährigen informellen Zusammenarbeit im Rahmen des SADCC mit der angestrebten politischen, sozialen und wirtschafulichen Integration emst meinten.

Im weiteren berichtete der Referent uber die Grunde, die zu der neuen Phase der intemationalen Kooperation im sudlichen Afrika auf der Basis eines völkerrechtlichen Vertrages geführt hätten. Entscheidend seien vor allem die stete Zunahme der Aufgaben der SADCC als Institution gewesen, die auf der informellen Gundlage schwerlich ausgeführt werden konnten, sowie Verändenungen in den regionalen sozio-ökonomischen Entwicklungen und der Druck nach einer rechtlichen Verfestigung, der von anderen internationalen Organisationen im suddichen Afrika auf die SADCC ausgeubt worden sei.

Anschließend erläuterte Mvungi den Aufbau des SADC-Vertrages, der sich in 18 Abschnitte mit 44 Artikel aufgliedert, und zeigte einige Aspekte des Abkommens auf, die die Struktur und die Aufgaben der SADC verdeutlichen. In seiner abschlieBenden Bewertung des SADC-Vertrages bemerkte der Referent, daB nicht etwa die juristischen Inhalte des Abkommens von besonderer Bedeutung für die Bevölkenung des südlichen Afrika, sondern vielmehr die Tatsache entscheidend sei, daB aufgrund der SADC erstmals der Versuch untemommen werde, die Völker des südlichen Afrika unter vielversprechenden Aussichten auf einen Wechsel der momentan miBlichen politischen, ökonomischen und sozialen Verhälmisse in dieser Region zu vereinen. Er beendete seine Ausfühnmgen insbesondere mit der Empfehlung, daB von der SADC Institutionen errichtet werden sollten, die sowohl auf die Verwirklichung wie auch auf die Wahrung von Menschenrechten, Demokratie und Rechtstaatlichkeit in den Mitgliedsstaaten hinwirken.

In der anschließenden Aussprache wurden insbesondere die juristischen Probleme erörtert, die bei der Vereinigung unterschiedlicher Rechts- und Wirtschaftssysteme der an der SADC teilhabenden Staaten aufzutreten pflegen, sowie ob und wie die westlichen Industriestaaten mit beratender "juristischer Entwicklungshilfe" zur Verwirklichung des Projekts beizutragen vermögen. 
Nach dieser Diskussion schloß Herr Prof. Dr. Ulrich Spellenberg die XIX. Jahrestagung und leitete zur Mitgliederversammlung der Gesellschaft für Afrikanisches Recht e.V. über, in deren Verlauf u.a. die tumusmäßigen Neuwahlen des Vorstandes 2 stattfanden und der Termin für die nächste Konf erenz auf den 4. und 5. November 1994 festgelegt wurde.

2 In den Vorstand der Gesellschaft für Afrikanisches Recht e.V. wurden folgende Mitglieder gewählt: Dr. Kurt Madlener (Freiburg), Prof. Dr. Wilhelm Möhlig (Köln), Prof. Dr. Hans-H. Münkner (Marburg), Prof. Dr. Heinrich Scholler (München), Prof. Dr. Ulrich Spellenberg (Bayreuth), Prof. Dr. Klaus Wähler (Berlin), Dr. Ulrike Wanitzek (Bayreuth). 